\title{
To Study Channel Estimation Techniques In Mimo Ofdm System
}

\author{
Deepika Sharma ${ }^{1}$, Rajiv Chechi ${ }^{2}$ \\ ${ }^{1,2}$ (ECED, HCTM TECHICAL CAMPUS KAITHAL-136027,HARYANA, INDIA)
}

\begin{abstract}
A multiple-input multiple output (MIMO) communication system is used with orthogonal frequency division multiplexing(OFDM) modulation technique to achieve high and reliable data rate transmission over broadband wireless channels. The need of MIMO OFDM is important because of only MIMO improves the system capacity without additional bandwidth.[1] The performance of MIMO-OFDM system is evaluated on the basis of Bit Error Rate(BER) and Mean Square Error(MSE). The correct channel estimation requires channel response of subcarriers between the pilot tones. Usually the received signal is distorted by channel characteristics. To recover the transmitted bits channel effects must be estimated. By orthogonality principle, each component of received subcarrier is to be expressed as product of transmitted signal and channel frequency response of subcarriers. So, the transmitted signal is recovered by estimating the channel response just at each subcarrier. Generally, data signal as well as training signal, or both, can be used for channel estimation. In this Paper, we will discuss channel estimation techniques in brief. The estimation of channel estimation technique will be carried out through MATLAB simulation.[3]
\end{abstract}

Keywords: Channel estimation, MIMO OFDM, Pilot carriers, PSAM, LS, MMSE, DFT based and DD Estimation Techniques.

\section{INTRODUCTION}

Orthogonal frequency division multiplexing (OFDM) is based on multicarrier communication techniques. The idea of multicarrier communications is to divide the total signal bandwidth into number of subcarriers. A disadvantage of the OFDM approach, is the increased complexity over the conventional system caused by employing $N$ modulators and filters at the transmitter and $N$ demodulators and filters at the receiver. However, this complexity can be removed by the use of the FFT and IFFT at the receiver and transmitter, respectively. The MIMO OFDM system consists of $\mathrm{Nt}$ transmit and $\mathrm{Nr}$ receiving antenna. Training symbols are used along with pilots in frequency domain.[2] MIMO OFDM is a new broadband wireless technology used in channel estimation due to high data rate and because of its robustness against multipath fading effects. The major problem faced in channel estimation is how to obtain the channel state information correctly. In training symbol based channel estimation the two types of arrangements are used along with the pilot symbol assisted modulation(PSAM) technique. One is the block type and the another is comb type, the block type arrangement channel estimation is done under the assumption of slow fading(channel transfer function not changes rapidly) while in comb type, the interpolation(linear and cubic spline) is used for the estimation.

The another techniques are LS(least square) and MMSE(minimum mean square estimation) are widely used for estimation. In the LS estimation, the estimation is done in such a way such that the cost function should be minimized. This method is simple to use. In MMSE, the estimation vector is used by orthogonality principle. This technique is better but more complex than LS estimation. In DFT based, effect of noise outside the maximum channel delay is eliminated and in this, performance is improved as compare to LS and MMSE estimation. In Decision Directed(DD) estimation, the coefficients of channel are updated after the initial estimation is made with preamble or pilots.[2] It uses the detected signal feedback to for tracking the time varying channel to detect the signal.

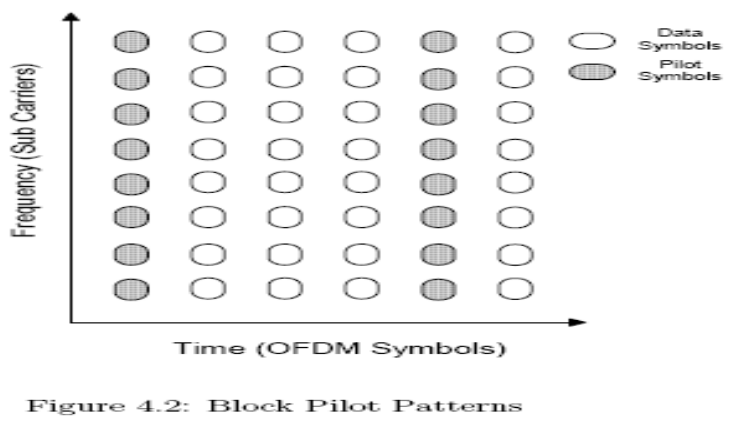

Figure 1 BLOCK Type Arrangement 


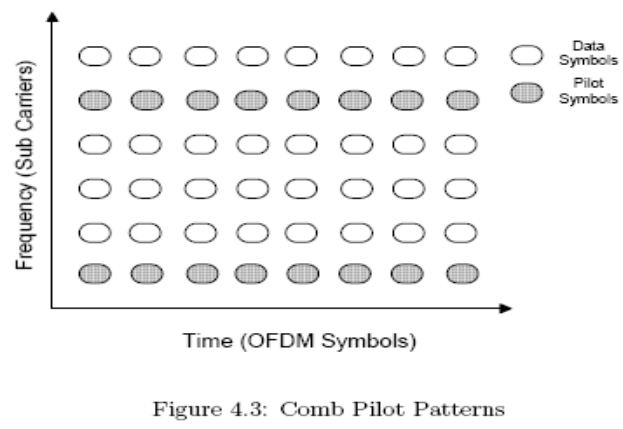

Figure 2 COMB Type arrangement

\section{PILOT SYMbol Assisted Modulation}

Channel estimation needs the multiplexing of pilot symbols into the data sequence, the technique is called as pilot symbol assisted modulation. This method depends upon the insertion of known phasors into the stream of useful information symbols for the purpose of channel estimation. These pilot symbols allow the receiver for extracting the channel attenuations of fading envelope and phase. Closed form formula for the BER of PSAM is provided for binary phase shift keying (BPSK) and quadrature phase shift keying (QPSK), while for 16-QAM is derived for a tight upper bound of the BER.A fading channel needs constant tracking, so pilot information is to be transmitted more or less rapidly. Decision directed channel estimation can also be used, but even in such type of schemes pilot information is to be transmitted regularly to mitigate error propagation.[2] Pilot symbols are transmitted at certain locations of the OFDM frequency time lattice, instead of data. Generally, fading channels can be viewed as in 2-D signal(time and frequency).

\section{LS ESTIMATION}

In the Least Square estimation the error between the measurements is minimized. If ' $\mathrm{X}$ ' is the transmitted symbol and ' $\mathrm{Y}$ ' is the received, then for ' $\mathrm{h}$ ' attenuations LS estimate is given by

$$
\hat{\mathbf{h}}_{\ell s}=\mathbf{X}^{-1} \mathbf{Y}=\left[\begin{array}{llll}
\frac{y_{0}}{x_{0}} & \frac{y_{1}}{x_{1}} & \ldots & \frac{y_{N-1}}{x_{N-1}}
\end{array}\right]^{T}
$$

\section{(Equation 1)}

Where $\mathrm{Np}$ is the number of pilots, pilot signal $\mathrm{Xp}(\mathrm{m}), \mathrm{m}=0,1 \ldots \ldots, \mathrm{Np}-1 . \mathrm{It}$ also finds the method to find the estimation of channel such that the cost function should be minimized.[3]

$$
\begin{aligned}
J(\hat{\mathbf{H}}) & =\|\mathbf{Y}-\mathbf{X} \hat{\mathbf{H}}\|^{2} \\
& =(\mathbf{Y}-\mathbf{X} \hat{\mathbf{H}})^{H}(\mathbf{Y}-\mathbf{X} \hat{\mathbf{H}}) \\
& =\mathbf{Y}^{H} \mathbf{Y}-\mathbf{Y}^{H} \mathbf{X} \hat{\mathbf{H}}-\hat{\mathbf{H}}^{H} \mathbf{X}^{H} \mathbf{Y}+\hat{\mathbf{H}}^{H} \mathbf{X}^{H} \mathbf{X} \hat{\mathbf{H}}
\end{aligned}
$$

\section{(Equation 2)}

By setting the derivative of the equation w.r.t. zero we get

$$
\frac{\partial J(\hat{\mathbf{H}})}{\partial \hat{\mathbf{H}}}=-2\left(\mathbf{X}^{H} \mathbf{Y}\right)^{*}+2\left(\mathbf{X}^{H} \mathbf{X H}\right)^{*}=0
$$

\section{(Equation 3)}

In this $\mathrm{X}$ is assumed to be as diagonal because of ICI free condition

$$
\hat{H}_{L S}[k]=\frac{Y[k]}{X[k]}, \quad k=0,1,2, \cdots, N-1
$$

(Equation 4)

Whereas the MEAN SQUARE ERROR is given by

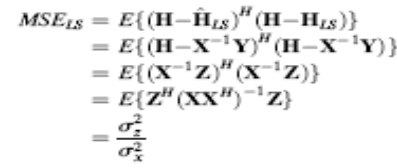

\section{(Equation 5)}


The above equation is proportional to SNR.[3]

\section{MMSE ESTIMATION}

In MMSE Estimation technique, we use weighted matrix and error is estimated as shown with the help of diagram

$$
J(\hat{\mathbf{H}})=E\left\{\|\mathbf{e}\|^{2}\right\}=E\left\{\|\mathbf{H}-\hat{\mathbf{H}}\|^{2}\right\}
$$

\section{(Equation 6)}

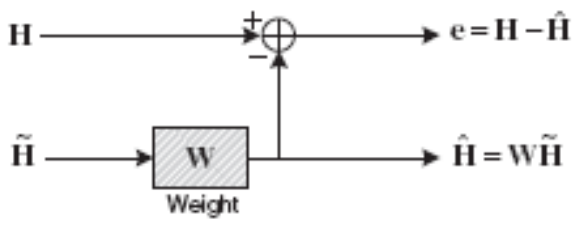

Figure 3 MMSE Estimation[3]

The estimation of error vector ' $\mathrm{e}$ ' is shown as

$$
\begin{aligned}
E\left\{\tilde{\mathbf{H}}^{H}\right\} & =E\left\{(\mathbf{H}-\hat{\mathbf{H}}) \tilde{\mathbf{H}}^{H}\right\} \\
& =E\left\{(\mathbf{H}-\mathbf{W} \tilde{\mathbf{H}}) \tilde{\mathbf{H}}^{H}\right\} \\
& =E\left\{\mathbf{H} \tilde{H}^{H}\right\}-\mathbf{W} E\left\{\hat{\mathbf{H}} \tilde{H}^{H}\right\} \\
& =\mathbf{R}_{\mathbf{H}}-\mathbf{W R}_{\tilde{\mathbf{H}} \tilde{\mathbf{H}}}=\mathbf{0}
\end{aligned}
$$

\section{(Equation 7)}

In weighted matrix autocorrelation and cross correlation function $\mathrm{s}$ are used, where ' $\mathrm{X}$ ' is the transmitted, ' $\mathrm{Y}$ ' is the received signal and $\mathrm{Z}$ is the noise vector introduced.

$$
\tilde{\mathbf{H}}=\mathbf{X}^{-1} \mathbf{Y}=\mathbf{H}+\mathbf{X}^{-1} \mathbf{Z}
$$

\section{(Equation 8)}

$$
\mathbf{W}=\mathbf{R}_{\mathrm{HH}} \mathbf{R}_{\mathrm{HH}}^{-1}
$$

\section{(Equation 9)}

$$
\begin{aligned}
\hat{\mathbf{H}} & =\mathbf{W} \hat{\mathbf{H}}=\mathbf{R}_{\mathbf{H H}} \mathbf{R}_{\hat{\mathrm{HH}}}^{-1} \hat{\mathbf{H}} \\
& =\mathbf{R}_{\mathbf{H H}}\left(\mathbf{R}_{\mathbf{H H}}+\frac{\sigma_{z}^{2}}{\sigma_{x}^{2}} \mathbf{I}\right)^{-1} \tilde{\mathbf{H}}
\end{aligned}
$$

\section{(Equation 10)}

Hence the estimated response in MMSE technique is shown by above equation.[3]

\section{DFT BASED ESTIMATION}

In DFT based estimation, the performance is improved as compare to LS and MMSE estimation.

$$
\operatorname{IDFT}\{\hat{H}[k]\}=h[n]+z[n] \triangleq \hat{h}[n], \quad n=0,1, \ldots, N-1
$$

(Equation 11)

By taking the IDFT of the channel $\mathrm{z}[\mathrm{n}]$ denotes the noise component in time domain and $\mathrm{h}[\mathrm{n}]$ is the response, the DFT is given by

$$
\hat{h}_{D F T}[n]= \begin{cases}h[n]+z[n], & n=0,1,2, \cdots, L-1 \\ 0, & \text { otherwise }\end{cases}
$$

If ' $L$ ' is the maximum channel delay then the procedural DFT based estimation is shown as ion figure below 


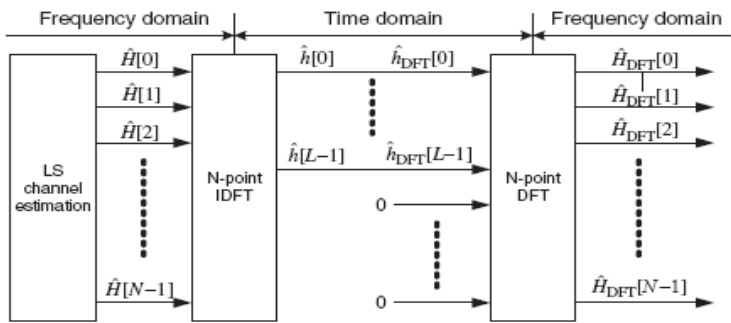

Figure 6.6 DFT-based channel estimation.

Figure 4 DFT Based Estimation[3]

\section{DECISION DIRECTED ESTIMATION}

This technique does not require the premable or pilots for estimation. The technique uses the detected signal feedback for continuously tracking the signal. The two steps are used from which step 2 follows from the symbol detection as done in step 1, the steps are given as

$$
\text { Step 1: } \hat{X}_{l}[k]=\frac{Y_{l}[k]}{\hat{H}_{l-1}[k]}
$$

Step 2: $\hat{H}_{l}[k]=\frac{Y_{l}[k]}{\bar{X}_{l}[k]}$

The OFDM receiver is linked with Decision Directed estimation is given as shown by diagram given below

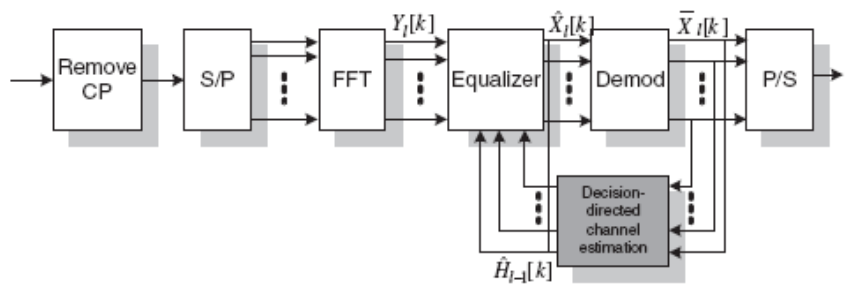

Figure 5 Decision Directed Estimation[3]

In this estimation if any error occurs in detected symbol then it is immediately propagated to degrade its performance. In this case the performance degradation is reduced as compare to previous techniques.[3]

\section{CONCLUSION}

In this paper different channel estimation techniques are discussed. The techniques are used for improving the performance. Pilot symbols are used with data symbols as described in Pilot Symbol Assisted Technique(PSAM). Firstly, the Least Square(LS) estimation is used which is very simple to implement so widely used. Then MMSE technique uses the weighted matrix to improve the performance but in this system is complex. In DFT based estimation DFT and IDFT are taken into the account along with the LS estimation to enhance the performance. Finally the Decision Directed technique feedback method is used to detect the error occurs during the transmission. In this technique the performance is improved up to certain desirable level. ICI and ISI problems are reduced with the help of these techniques.

\section{Examples follow:}

\section{References}

\section{Journal Papers:}

[1] MM Rana "Channel estimation techniques and LTE Terminal implementation challenges" in Proc. International Conference on Computer and Information Technology pp. 545-549 December 2010

[2] M. Simko, D. Wu, C. Mehlführer, J. Eilert, D. Liu "Implementation Aspects of Channel Estimation for 3GPP LTE Terminals" in Proc. Proc. European Wireless 2011.

Books:

[3] MIMO OFDM Wireless communications with MATLAB by Yong Soo Cho\Jaekwon Kim Won Young Yang/Chung-Gu Kang. Theses:

[4] Michall David Larsen, Studies o the Performance and Impact of channel estimation in MIMO and OFDM System, Brigham Young University,2010 . 